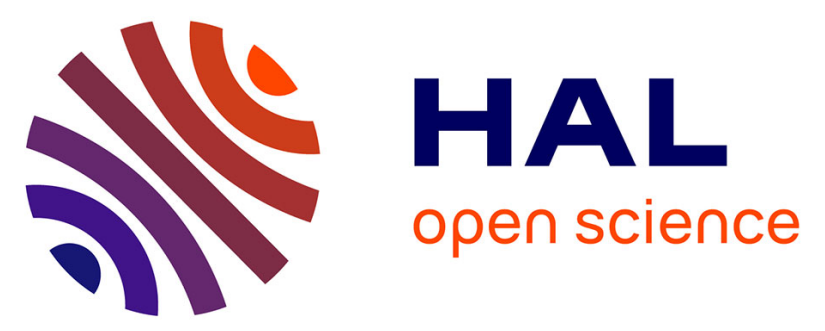

\title{
Impact de la variabilité non-mesurée des précipitations sur les débits en hydrologie urbaine: un cas d'étude dans le cadre multifractal
}

\author{
Auguste Gires, D Schertzer, Ioulia Tchiguirinskaia, S. Lovejoy, Cedo \\ Maksimovic, Christian Onof, Nuno Simoes
}

\section{To cite this version:}

Auguste Gires, D Schertzer, Ioulia Tchiguirinskaia, S. Lovejoy, Cedo Maksimovic, et al.. Impact de la variabilité non-mesurée des précipitations sur les débits en hydrologie urbaine: un cas d'étude dans le cadre multifractal. La Houille Blanche - Revue internationale de l'eau, 2011, 4 (sept 2011), pp.37-42. 10.1051/lhb/2011039 . hal-00677434

\section{HAL Id: hal-00677434 \\ https://hal-enpc.archives-ouvertes.fr/hal-00677434}

Submitted on 24 May 2012

HAL is a multi-disciplinary open access archive for the deposit and dissemination of scientific research documents, whether they are published or not. The documents may come from teaching and research institutions in France or abroad, or from public or private research centers.
L'archive ouverte pluridisciplinaire HAL, est destinée au dépôt et à la diffusion de documents scientifiques de niveau recherche, publiés ou non, émanant des établissements d'enseignement et de recherche français ou étrangers, des laboratoires publics ou privés. 


\title{
Impact de la variabilité non-mesurée des précipitations sur les débits en hydrologie urbaine : un cas d'étude dans le cadre multifractal
}

\author{
Auguste GIRES ${ }^{(1)}$, Daniel SCHERTZER ${ }^{(1)}$, Ioulia TCHIGUIRINSKAIA ${ }^{(1)}$, Schaun LOVEJOY(2), \\ Cedo MAKSIMOVIC ${ }^{(3)}$, Christian ONOF $^{(3)}$, Nuno SIMOES ${ }^{(3,4)}$ \\ (1) Université Paris-Est Ecole des Ponts ParisTech LEESU, 6-8 Av Blaise Pascal Cité Descartes, Marne-la-Vallee, 77455 Cx2, France - e-mail : \\ auguste.gires@leesu.enpc.fr \\ (2) McGill U., Physics dept., Montreal, PQ, Canada \\ (3) Imperial College London, Dept of Civil and Environmental Engineering, United-Kingdom \\ (4) Department of Civil Engineering, University of Coimbra, Coimbra, Portugal
}

\begin{abstract}
RÉSUMÉ. - Cet article utilise les propriétés multifractales d'un évènement pluvieux dans la région de Londres le 9 février 2009, pour mieux comprendre et quantifier l'incertitude associée à la variabilité spatio-temporelle des précipitations non mesurées par les radars météorologiques en bande $C$, dont la résolution estimée est de $1 \mathrm{~km} * 1 \mathrm{~km} * 5 \mathrm{~min}$, et comment elle se transfère aux prévisions des débits en réseaux d'assainissement. Le cas d'étude hydrologique est celui du bassin versant urbain de 900 hectares de Cranbrook (Londres). Les propriétés multifractales sont en accord avec le modèle spatio-temporel le plus simple, reposant sur un exposant d'anisotropie entre l'espace et le temps. Ceci permet de désagréger le champ de précipitation à l'aide de cascades multifractales spatio-temporelles. Un ensemble de champs de précipitations désagrégés réalistes est alors généré à l'aide des multifractals universels, puis l'ensemble des hydrogrammes correspondants est obtenu par un modèle urbain pluie-débit semi-distribué. Il apparait que les queues de probabilité issues de l'analyse de 100 échantillons de précipitation présentent un comportement en loi de puissance, qui est retrouvé sur les débits de pointe mais avec des exposants différents
\end{abstract}

Mots-clés : variabilité spatio-temporelle, désagrégation, multifractals, loi de puissance, ensemble stochastique, hydrologie urbaine.

\section{Impact of unmeasured rainfall variability on urban discharge : a case study in a multifractal framework}

\begin{abstract}
In this paper, the multifractal properties of a rainfall event, that occurred over the London area on February 9th 2009, are used to better understand and quantify the uncertainty associated with the rainfall variability not resolved by C-band radar, whose resolution is estimated at $1 \mathrm{~km} * 1 \mathrm{~km} * 5 \mathrm{~min}$, and how it is transferred to sewer discharge. The hydrological case study is the 900 ha urban catchment of Cranbrook (London). The multifractal properties of the rainfall field are in agreement with the simplest case of space-time multifractals that relies on a unique anisotropy exponent between space and time. This enables a multifractal downscaling of the rainfall field. First an ensemble of realistic downscaled rainfall fields is generated with the help of Universal Multifractals. Then the corresponding ensemble of hydrographs is simulated with the help of a semi-distributed urban model. It appears that the tail of probability distribution of the rainfall maximum (among the 100 samples generated) exhibit a power-law behaviour. The same statistical behaviour is observed for peak flows, but with different characteristic exponents.
\end{abstract}

Key-words : spatio-temporal variability, downscaling, multifractals, power-law, stochastic ensembles, urban hydrology.

\section{INTRODUCTION}

Un mode classique de représentation, en relation avec la notion de cascade ([1] pour une revue récente) conduit à analyser et simuler l'extrême variabilité des processus géophysiques sur de grandes gammes d'échelle, en particulier la pluie, dans le cadre des multifractals universels, à l'aide d'un nombre réduit de paramètres [2,3]. De telles analyses menées sur les débits et/ou les précipitations, ont permis d'éclairer la compréhension de la relation pluie-débit en comparant leurs paramètres multifractals respectifs $[4,5,6,7]$ ainsi que le comportement de leurs extrêmes [8]. Par ailleurs, de nombreuses études d'hydrologie plus classiques $[9,10,11,12,13]$ suggèrent un impact significatif de la variabilité spatio-temporelle sur les débits même si des nuances sont à apporter en fonction du type d'événements pluvieux, de la taille du bassin versant et de ses caractéristiques. En hydrologie urbaine, avec des bassins versants plus petits et une proportion plus grande de pluie efficace en raison de coefficients d'imperméabilisation supérieurs, les effets semblent plus importants $[14,15]$.

Cet article met en œuvre les méthodes multifractales en hydrologie urbaine afin de mieux comprendre comment l'incertitude sur la variabilité spatio-temporelle à très haute résolution de la pluie (i.e. au-delà de la résolution de $1 \mathrm{~km} * 1 \mathrm{~km} * 5 \mathrm{~min}$ classiquement disponible avec les réseaux radars à bande $\mathrm{C}$ des services météorologiques) se reflète dans les résultats de simulation d'un modèle semi-distribué d'hydrologie urbaine. Le cas d'étude est celui du bassin versant de Cranbrook une zone urbaine de 900 hectares située 
dans l'Est de Londres, et déjà utilisée dans [16]. Dans un premier temps, les propriétés multifracales spatio-temporelles de l'évènement de la région londonienne du 9 février 2010 sont analysées. Ensuite, une démarche reposant sur des ensembles stochastiques est adoptée: (i) Un ensemble de champs réalistes de précipitations désagrégées à la résolution $111 \mathrm{~m} * 111 \mathrm{~m} * 1.25 \mathrm{~min}$ est généré à l'aide de cascades multifractales universelles; (ii) Les hydrogrammes correspondants sont simulés. Ces ensembles de réalisation permettent enfin de caractériser l'incertitude d'abord sur les pluies puis sur les débits de pointe. Cet article s'intéresse principalement aux échantillons extrêmes générés, c'est-à-dire aux queues de probabilité

\section{QUANTIFICATION DE LA VARIABILITE SPATIO-TEMPORELLE DE LA PLUIE}

\section{II.1. Les données de pluie}

Les données de pluie utilisées dans cette étude sont issues des mosaïques radar Nimrod, un produit du UK Meteorological Office. Le traitement Nimrod des données radar contient principalement une interpolation des données issues de tous les radars à bande $\mathrm{C}$ du réseau britannique, des corrections à base physique, et un ajustement avec le réseau de pluviomètres [17]. La résolution affichée des données Nimrod est de $1 \mathrm{~km}$ en espace et 5 min en temps. Une zone de $64 \mathrm{~km} * 64 \mathrm{~km} * 21 \mathrm{~h}$ centrée sur la partie la plus intense de l'évènement du 9 février a été analysée. Une carte de la hauteur totale précipitée est en Fig. 1a.

\section{II.2. Analyse spatio-temporelle multifractale}

Pour un champ multifractal stochastique les moments statistiques d'ordre $q$ du champ de précipitation $R_{\lambda}$ à la résolution $\lambda(=L / l$, le rapport entre l'échelle extérieure et celle d'observation du phénomène) présente le comportement scalant suivant $(<.>$ désigne l'espérance mathématique ou moyenne d'ensemble) :

$$
<R_{\lambda}^{q}>=\lambda^{K(q)}<R_{1}^{q}>
$$

où $K(q)$ est la fonction des moments d'échelle. Cette expression n'est a priori valable qu'en moyenne sur un ensemble infini de réalisations, Ici deux types d'analyse en moyenne d'ensemble sont menées : une analyse spatiale (une réalisation est alors le champ 2D observé à un chaque pas de temps) et une temporelle (une réalisation est alors la série temporelle $1 \mathrm{D}$ observée à chaque pixels radar). Dans le cadre des multifractals universels [2,3], la fonction $K(q)$ ne dépend que de trois paramètres $C_{1}$, $\alpha$, et $H . C_{1}$ mesure l'intermittence moyenne $\left(C_{1}=0\right.$ pour un champ homogène), $\alpha$ la multifractalité ( $\alpha=0$ pour un champ monofractal et $\alpha=2$ pour le cas limite log-normal), et $H$ la non-conservation ( $H=0$ pour un champ conservatif). $K(q)$ est alors donné par l'expression :

$$
K(q)=\frac{C_{1}}{\alpha-1}\left(q^{\alpha}-q\right)+H q,
$$

Les paramètres multifractals sont estimés grâce à l'analyse de la pente spectrale et à la méthode DTM [18].

La Fig. $1 \mathrm{~b}$ représente l'Eq. 1 dans un graphique en échelle log-log pour l'analyse spatiale. Il convient de préciser que pour tenir compte du fait que le champ observé n'est pas conservatif (on trouvera $H=0.56$ ) cette courbe a été tracée pour les fluctuations du champ et non pas le champ directement. Les lignes droites (coefficient R2 de 0.99 en moyenne) reflètent un comportement scalant, au moins sur la gamme d'échelle allant de 1 à $64 \mathrm{~km}$. Les pentes sont égales à $K_{\text {espace }}(q)$. Les valeurs de $\alpha, C_{1}$ et $H$ valent respectivement $1.62,0.14$ et 0.56 .

Une courbe similaire à celle de la Fig. $1 \mathrm{~b}$ mais en analyse temporelle confirme la présence d'un comportement scalant (coefficient R2 allant de 0.98 à 0.99 pour les $q$ compris entre 0.8 et 2.5 ) pour une gamme d'échelle allant de $5 \mathrm{~min}$ à $11 \mathrm{~h}$. Les estimations des paramètres $\alpha, C_{1}$ et $H$ valent respectivement $1.52,0.10$ et 0.21 . Ces résultats sont en accord avec le cadre du modèle spatio-temporel unifié et scalant le plus simple de pluie $[19,20,21]$, qui se base sur l'hypothèse d'un exposant d'anisotropie entre l'espace et le temps. Ce dernier suggère que les fonctions d'échelle des moments spatiale et temporelle devraient être proportionnelles, c'est-à-dire

$$
K_{\text {espace }}(q)=\frac{K_{\text {temps }}(q)}{1-H_{+}}
$$

Où $H_{t}$ est l'exposant d'anisotropie entre l'espace et le temps. Ceci implique des $\alpha$ identiques et des $C_{1}$ et $H$ présentant le même rapport. (a)

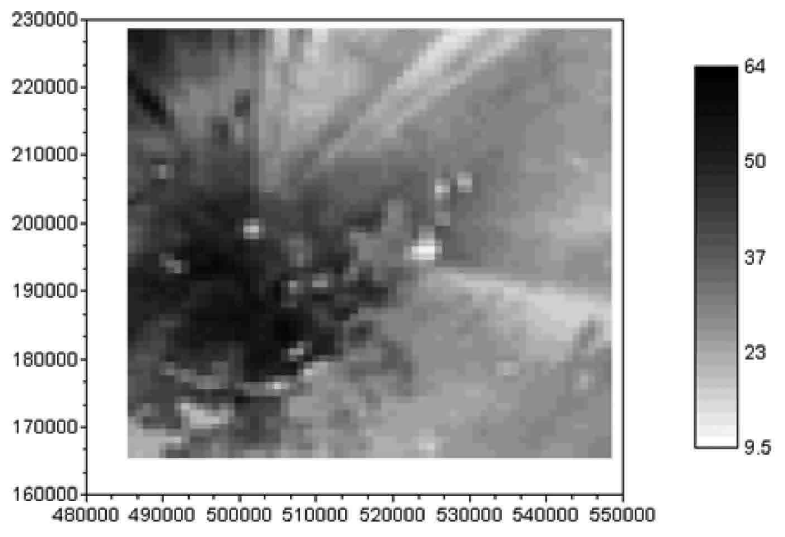

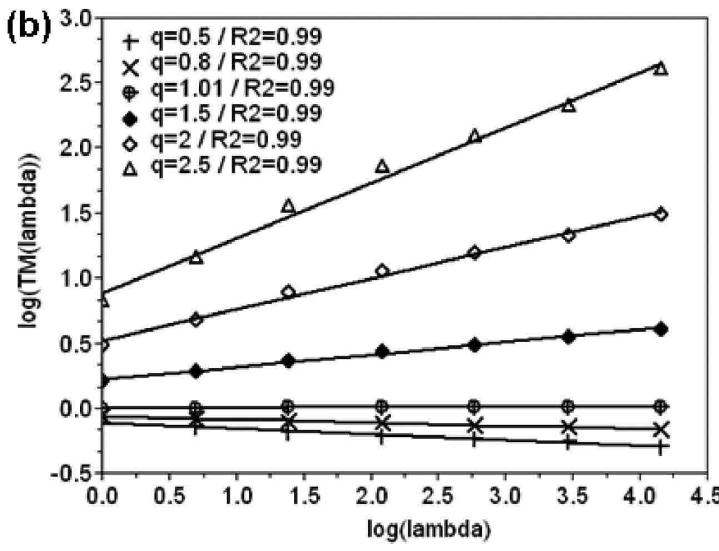

Figure 1: (a) Hauteur totale précipitée (en $\mathrm{mm}$ ) sur la zone d'étude pour l'évènement considéré. Le système de coordonnées est le «British National Grid» (http ://www.ordnancesurvey.co.uk/). (b) Illustration du comportement scalant (eq. 1 dans un graphique log-log) en analyse spatiale. 


$$
\frac{C_{1, \text { espace }}}{C_{1, \text { temps }}}=\frac{H_{\text {espace }}}{H_{\text {temps }}}=\frac{1}{1-H_{t}},
$$

Ici, les $\alpha$ sont proches et les rapports des $C_{1}$ et des $H$ correspondent à un $H_{\mathrm{t}}$ de respectivement 0.29 et 0.62 , ce qui est proche (surtout pour $C_{1}$ dont les estimations sont plus fiables) de la valeur théorique de $1 / 3$ obtenue avec la théorie de Kolmogorov en considérant la pluie comme un scalaire passif [19].

\section{CARACTERISATION DE L'INCERTITUDE SUR LA PLUIE ASSOCIEE A LA VARIABILITE NON MESUREE DE LA PLUIE}

\section{III.1. Désagrégation spatio-temporelle du champ de pluie}

En l'absence de mesure de pluie à très haute résolution (environ $100 \mathrm{~m}$ ) il est classique d'utiliser une désagrégation stochastique $[20,22,23,24,25]$. Le cadre des cascades est particulièrement adapté à cet exercice [26]. En effet la désagrégation consiste alors simplement à poursuivre stochastiquement la cascade au-delà de l'échelle d'observation. Plus précisément, pour chaque pixel 2 étapes de cascade spatiotemporelle [19,27], avec les paramètres $\alpha$ et $C_{1}$ estimés sur la gamme d'échelle allant de 1 à $64 \mathrm{~km}$, sont réalisées. Pour le modèle spatio-temporel, dont la pertinence a été validée sur cet évènement dans la section précédente, lorsque la résolution spatiale est divisée par $\lambda_{x}$, la résolution temporelle est divisée par $\lambda_{\mathrm{t}}=\lambda_{\mathrm{x}}{ }^{1-\mathrm{Ht}}$. Ainsi à chaque étape d'un tel processus un pixel-parent est divisé en 18 pixels-enfants, en divisant la résolution spatiale par 3 et la résolution temporelle par $2\left(2^{1-1 / 3} \approx 2,08\right)$. La valeur affectée à chacun des pixelsenfants est celle du pixel-parent multipliée par un coefficient aléatoire permettant de respecter les équations 1 et 2 . Ce processus, illustré en Fig. 2a permet de passer d'une résolution de $1 \mathrm{~km} * 1 \mathrm{~km} * 5 \mathrm{~min}$ à $111 \mathrm{~m} * 111 \mathrm{~m} * 1.25 \mathrm{~min}$. La valeur moyenne d'une sous-cascade est égale à un en moyenne d'ensemble. Un exemple de désagrégation pour un pas de temps est donné en Fig. 2b. La même palette de couleur a été utilisée pour l'image radar et le champ désagrégé afin de pouvoir les comparer. L'effet de la désagrégation qui génère des intensités plus ou moins fortes au sein d'un pixel radar est visible, particulièrement dans la zone de forte intensité au sud. Il convient de noter que si les désagrégations de deux pas de temps successifs sont indépendantes (i.e., les cascades générées pour chaque sous-pixel sont effectivement indépendantes), il n'en reste pas moins que les valeurs désagrégées restent dépendantes du fait que les structures à plus grandes échelles le sont. Nous nous sommes contentés dans ce papier de cascades discrètes (rapport d'échelle élémentaire $\lambda_{x}$ ), alors qu'en toute rigueur il faudrait recourir à des cascades continues pour respecter la causalité [3].

\section{III.2. Caractérisation de l'incertitude sur la pluie}

A partir des mêmes données radar à la résolution $1 \mathrm{~km} * 1 \mathrm{~km} * 5 \mathrm{~min}$, cent échantillons de pluie réaliste à plus haute résolution ont été générés pour mettre en œuvre une approche de type prévision d'ensemble. L'incertitude sur la pluie du fait de la limite de résolution du radar est caractérisée par la variabilité observée au sein de l'ensemble d'échantillons. Avant de poursuivre, il convient de mentionner que la hauteur totale moyenne de précipitation est égale à 19.14 $\pm 0.04 \mathrm{~mm}$ sur les différents échantillons, sachant que la valeur obtenue avec les données radar est de $19.14 \mathrm{~mm}$. Ceci signifie que la désagrégation, bien que non strictement conservative, ne perturbe que très légèrement l'accumulation totale de la pluie et donc que les effets hydrologiques observés sont dus à des différences de répartition spatiale. Dans la suite, les hydrogrammes de dix canalisations sont analysés. La racine carrée de la surface drainée par une canalisation définit la longueur charactéristique de son bassin versant associé $L_{\mathrm{sd}}$. La première étape consiste à définir pour chacun des échantillons de pluie et chaque canalisation une valeur particulière de la pluie. Deux types d'extrêmes du champ de précipitation ont été choisis dans cet article pour mener l'analyse :

- l'intensité désagrégée maximale obtenue sur la surface drainée par la canalisation et sur tout l'événement. Cette valeur est dénommée «pluie maximale » dans la suite.

- l'intensité maximale sur tout l'événement de l'intensité moyenne évaluée sur la zone drainée par la canalisation pour chaque pas de temps. Cette valeur est dénommée «pluie moyenne maximale » dans la suite.

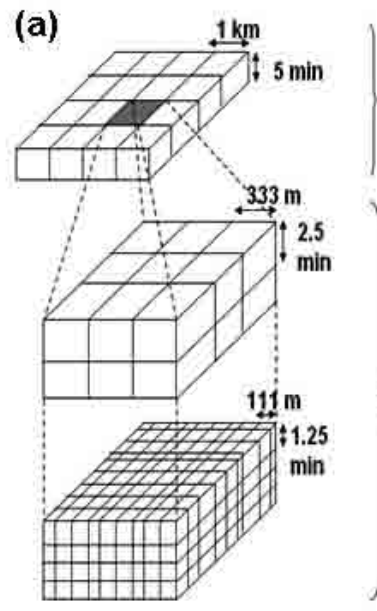

\section{Mesuré ou évalué déterministiquement \\ Analyse multifractale $\rightarrow \alpha$ and $C_{1}$}

\section{Désagrégation stochastique pour chaque pixels}

Faite à l'aide d'une cascade multifractale universelle discrète de paramètres $\alpha$ and $C_{1}$ (b)
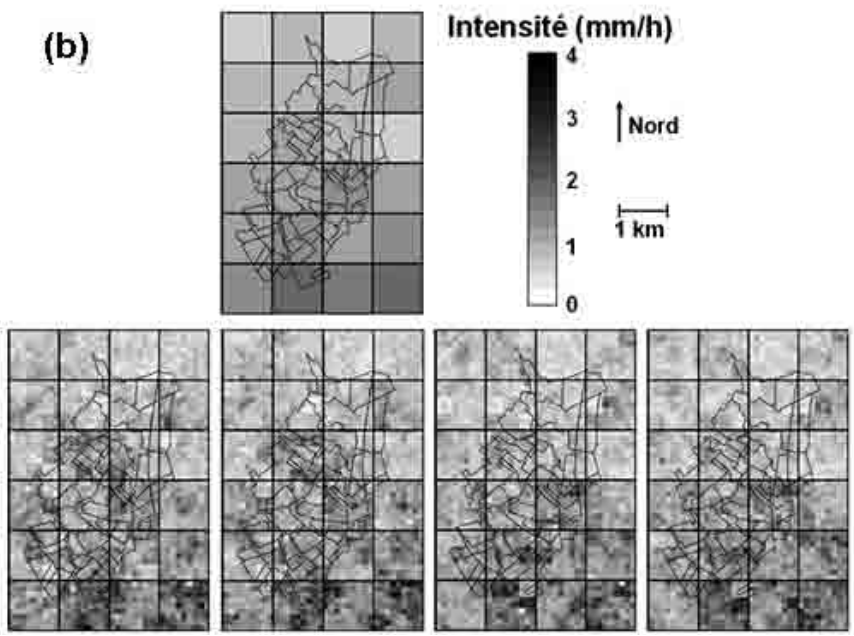

Figure 2 : Illustration (a) et exemple sur la zone de Cranbook (b) du processus de désagrégation spatio-temporelle pour un pas de temps. L'image radar de résolution spatiale $1 \mathrm{~km}$ (haut) est remplacée par 4 images successives (bas) possédant une résolution spatiale de $111 \mathrm{~m}$. 

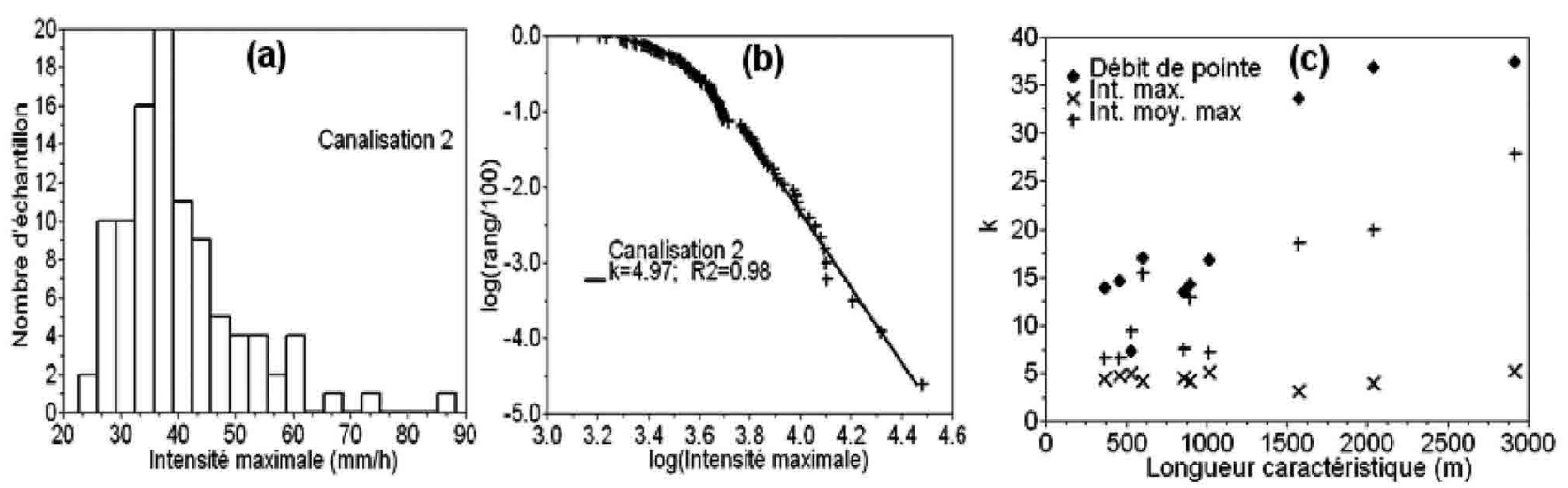

Figure 3 : Histogrammes (a) et mise en évidence (en coordonnées logarithmiques) de son comportement en loi de puissance (b) pour les 100 échantillons de précipitation pour la canalisation 2 (pluie maximale). (c) Exposant $k$ de la loi de puissance pour l'intensité maximale de la pluie, l'intensité moyenne maximale, et le débit de pointe en fonction de l'échelle du sous-bassin $L_{s d '}$

Une fois définie une valeur par échantillon et par canalisation, il est possible de tracer les histogrammes correspondants. En Fig. 3a ce trouve celui pour la pluie maximale de la canalisation 2 (qui est au sud du bassin versant, proche des zones d'intensité pluvieuse maximale). Les courbes obtenues pour les autres canalisations et la pluie moyenne maximale sont similaires. Une première étape pour caractériser l'histogramme de la Fig. 3.a consiste à estimer un coefficient de variation $\left(C V^{\prime}\right)$ défini de la façon suivante :

$$
C V^{\prime}=\frac{X_{0.95}-X_{0.05}}{2 * X_{0.5}}
$$

à partir des quantiles à $5\left(X_{0.05}\right)$ et $95 \%\left(X_{0.95}\right)$, et de la médiane $\left(X_{0.5}\right)$. On trouve que $C V$, varie de 33 à $60 \%$ pour la pluie maximale et de 24 à $38 \%$ pour la pluie moyenne maximale. Dans cet article nous nous intéressons plus spécifiquement aux échantillons extrêmes ; i.e. la partie droite des histogrammes. On constate qu'elles sont caractérisées par un comportement en loi de puissance, c'est-à-dire que l'on a :

$$
\operatorname{Pr}(X>x) \alpha x^{-k}
$$

où $k$ est l'exposant caractéristique. Afin de vérifier l'ajustement d'une loi de puissance, les intensités (moyennes) maximales simulées pour chaque échantillon ont été triées par ordre décroissant, et l'intensité en fonction du rang divisée par le nombre d'échantillon est tracée dans un graphique en échelle log-log. La Fig 3 b représente cette courbe pour la pluie maximale de la canalisation 2, et est représentative des autres. La ligne droite observée pour les grandes intensités reflète la loi de puissance sous-jacente. Les coefficients de détermination des régressions linéaires valent en moyenne (sur les 10 canalisations) 0,98 pour l'intensité maximale et 0,97 pour l'intensité moyenne maximale ce qui valide la caractérisation par une loi de puissance.

Les valeurs numériques des $k$ trouvées pour la pluie maximale $\left(k_{\text {pluie } \max }\right)$ et la pluie moyenne maximale $\left(k_{\text {pluie moy max }}\right)$ sont indiquées en Fig. 3c ainsi que celles des exposants trouvés pour les débits (cf. section suivante). Une valeur élevée de $k$ reflète des queues de probabilité moins épaisses et donc des extrêmes moins prononcés. On n'observe pas de forte dépendance de $k_{\text {pluie max }}$ avec $L_{\text {sd }}$, ce qui reflète que la pluie possède des propriétés statistiques comparables aux différentes échelles d'observation. En revanche $k_{\text {pluie moy max }}$ a tendance à augmenter avec $L_{\text {sd }}$, ce qui signifie, comme on pouvait s'y attendre, que plus la surface drainée par la cana- lisation est grande, plus l'effet de la variabilité non-mesurée de la pluie est amorti. On retrouve naturellement que $k_{\text {pluie max }}$ est inférieur à $k_{\text {pluie moy max }}$, puisque la moyenne faite dans l'analyse de l'intensité moyenne maximale amortie les extrêmes observés dans l'analyse de l'intensité maximale brute.

\section{TRANSFERT DES INCERTITUDES SUR LE DEBIT}

\section{IV.1. Présentation du modèle pluie-débit}

Le cas d'étude analysé dans cet article reprend celui de [16], dans lequel il est décrit avec plus de précision. Il correspond au bassin versant de versant de Cranbrook, à Redbridge (Londres, Royaume-Unis), un bassin essentiellement urbain d'environ 900 hectares, connu pour être un lieu fréquent d'inondations urbaines. Le modèle pluie-débit semidistribué utilisé a été fourni et calibré par Thames Water Utilities Ldt [28]. Le modèle décompose le bassin versant de Cranbrook en 51 sous-bassins considérés comme homogènes (de 1 à 62 hectares, avec des temps de concentration de l'ordre de quelques minutes à une dizaine de minutes) et prend en compte le réseau d'assainissement principal (Fig. 4). Les simulations sont réalisées à l'aide du logiciel d'hydrologie urbaine Infoworks CS [29]. Le paramétrage du modèle n'a pas été changé dans cette étude. La répartition spatiale du cumul total de pluie (via des images radar) au cours de l'évènement pluvieux est présentée en Fig. 4. Elle varie de 16 à $23 \mathrm{~mm}$ en fonction du sous-bassin versant avec d'avantage de pluie au sud, à proximité de l'exutoire. Dans cette étude les hydrogrammes de 10 canalisations sont analysés. Elles ont été sélectionnées de façon à couvrir une large gamme de $L_{\text {sd }}$. Ici $L_{\text {sd }}$ varie de 370 à $2910 \mathrm{~m}$ (Fig. 4).

\section{IV.2. Caractérisation des incertitudes sur les débits de pointe}

Pour l'ensemble des simulations de pluie désagrégée, les hydrogrammes des canalisations sont calculés. L'objet de cette section est d'analyser la variabilité au sein de l'ensemble d'hydrogrammes simulés et de comprendre en quoi elle reflète celle des pluies. On s'intéresse au débit de pointe, une grandeur fondamentale dans le dimensionnement et la gestion des réseaux d'assainissement. Pour chaque échantillon le débit 


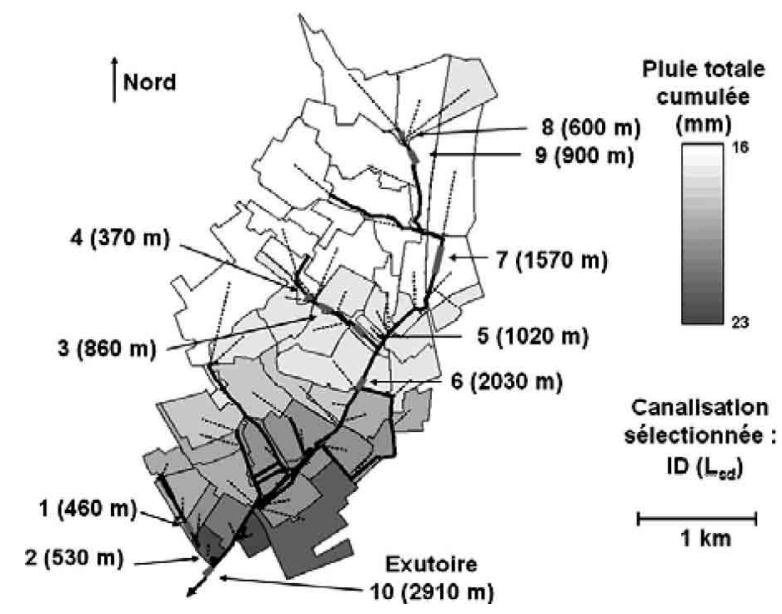

Figure 4: Le bassin versant de Cranbrook, et son réseau d'assainissement modélisé, ainsi que le cumul de précipitation au cours de l'évènement pluvial pour chaque sousbassin versant.

de pointe et son heure d'occurrence ont été estimés. Aucune influence significative n'est observée sur les heures d'occurrence, si bien qu'aucune étude supplémentaire n'est faite dans la suite. Pour les débits de pointe, la même analyse que pour les précipitations est faite. Le coefficient $C V^{\prime}$ vaut de 5 (à l'aval) à $26 \%$ (à l'amont). Des courbes similaires à celles en Fig. 3a (histogramme des valeurs) et $3 b$ (courbe de détermination de l'exposant $k$ de la loi de puissance) sont obtenues. Les coefficients de détermination des régressions linéaires valent en moyenne (sur les 10 canalisations) 0,98 ce qui valide la caractérisation par une loi de puissance. Les exposants $k\left(k_{\text {débit }}\right)$ sont présentés Fig. 3c.

Il est notable de constater que la relation pluie-débit conserve le comportement statistique en loi de puissance observé sur les précipitations, mais en change les exposants caractéristiques. L'observation de loi de puissance signifie que les queues de probabilité associées à la variabilité spatio-temporelle non mesurée à petite échelle sont épaisses et ne peuvent donc pas être négligées. Ceci implique qu'elles doivent être prises en compte dans le dimensionnement et la gestion des réseaux d'assainissement. Il est remarquable de noter que malgré la faible résolution spatiale du modèle pluie-débit (en moyenne la racine carrée de l'aire des sousbassins versant vaut $380 \mathrm{~m}$ ) par rapport à la résolution des précipitations désagrégées $(111 \mathrm{~m})$, qui fait qu'une partie de l'information issue de la désagrégation est perdue, et de probables limitations dans la modélisation pluie-débit, les incertitudes associées à la variabilité non mesurée de la pluie sont significatives.

On retrouve pour l'exposant $k_{\text {débit }}$ la croissance avec $L_{\text {sd }}$ observée pour $k_{\text {pluie moy max }}$, et les valeurs numériques sont globalement supérieures pour le débit, mais restent comparables. En revanche $k_{\text {pluie maximale }}$ est inférieur à $k_{\text {débit }}$ pour toutes les canalisations. Ceci semble montrer que les débits ne reproduisent pas les extrêmes de la pluie maximale, mais ceux de la pluie moyenne maximale. Ces derniers sont assez peu affectés par le passage en réseau. Pour obtenir une analyse plus fine des résultats, il conviendrait de mener de futures études avec un modèle pluie-débit possédant une meilleure résolution spatiale et prenant en compte les interactions entre les flux de surface et les flux dans les canalisations [30,31] pour profiter pleinement de la désagrégation du champ de précipitation.

\section{CONCLUSION}

La première conclusion de cette étude est que les données radar de l'événement pluvieux du 9 février 2010 de la région londonienne sont en accord avec le modèle spatiotemporel scalant le plus simple reposant sur un exposant d'anisotropie entre l'espace et le temps. Cet accord a permis la mise en place d'une désagrégation spatio-temporelle réaliste du champ de précipitations. L'approche reposant sur des ensembles stochastiques mise en œuvre a permis de quantifier l'incertitude associée à la variabilité spatiotemporelle non-mesurée des précipitations, et comment elle se transfère aux hydrogrammes du réseau d'assainissement du bassin versant urbain de Cranbrook (Londres). Il apparait que la distribution de probabilités des extrêmes de la pluie désagrégée est en loi de puissance. Ce type de loi est retrouvé pour les débits de pointe simulés, ce qui par ailleurs signifie que l'incertitude associée à la variabilité non mesurée du champ de pluie ne peut pas être négligée dans la gestion et le dimensionnement des réseaux d'assainissement. Cela souligne l'intérêt d'implanter des radars à bande $\mathrm{X}$ en milieu urbain, puisqu'ils permettent d'obtenir une résolution spatiale de l'ordre de $100 \mathrm{~m}$. En revanche, l'établissement d'un lien clair entre les exposants des lois de distribution des extrêmes des précipitations et des débits nécessite l'analyse de davantage de cas d'étude et d'événements pluvieux. Par ailleurs, cette étude ouvre une piste pour tester autrement les modèles hydrologiques, en s'assurant qu'ils sont capables à partir de différentes pluies extrêmes de reproduire le comportement statistique observé sur les débits associés.

\section{REMERCIEMENTS}

A. Gires remercie 1'Université Paris-Est pour son soutien financier. Il remercie également l'Imperial College London pour son soutien financier partiel. A. Gires, I. Tchiguirinskaia et D. Schertzer remercient la chaire «Hydrologie pour une ville résiliente» de l'Ecole des Ponts ParisTech en partenariat avec Véolia, pour son soutien financier. Les auteurs remercient MWH Soft, Thames Water Utilities Ltd et le UK Met Office pour les données. N. Simões remercie la Fundação para a Ciência e Tecnologia - Ministério para a Ciência, Tecnologia e Ensino Superior, Portugal [SFRH/ BD/37797/2007] pour son soutien financier.

\section{REFERENCES}

[1] Lovejoy S., \& Schertzer D. (2007) - Scale, scaling and multifractals in geophysics : twenty years on. Nonlinear dynamics in Geosciences. Ed. A.A. Tsonis, J. Elsner, Springer. 311337

[2] Schertzer D., \& Lovejoy S. (1987) - Physical modelling and analysis of rain and clouds by anisotropic scaling and multiplicative processes. J. Geophys. Res. 92(D8) 9693-9714

[3] Schertzer D., Lovejoy S., Schmitt F., Tchiguirinskaia I., \& MARSAN D. (1997) - Multifractal cascade dynamics and turbulent intermittency. Fractals. 5(3) 427-471

[4] Tessier Y., Lovejoy S., Hubert P., Schertzer D., \& PeCKNOLdL D. (1996) - Multifractal analysis and modeling of rainfall and river flows and scaling, causal transfer functions. J. Geophys. Res. 101(D21) 26, 427-440

[5] Pandey G., Lovejoy S., \& Schertzer D. (1998) Multifractal analysis including extremes of daily river flow 
series for basins one to a million square kilometres. J. Hydrol. 208(1-2) 62-81

[6] LABAT D., Mangin A., \& ABABou R. (2002) - Rainfall-runoff relations for karstic springs : multifractal analyses. J. Hydrol. 256 176-195

[7] Tchiguirinskaia I., Schertzer D., Hubert P., Bendjoudi H., \& LoveJoY S. (2007) - Potential of multifractal modelling of ungauged basins. PUB Kick-Off Meeting. D. Schertzer et al. Wallingford, UK, IAHS Press. 309 298-308

[8] Schertzer D., Bernardara P., Biaou A., Tchiguirinskaia I., Lang M., Sauquet E., Bendjoudi H., Hubert P., Lovejoy S., \& Veysseire J.M. (2006) - Extrêmes et multifractals en hydrologie : résultats, validations et perspectives. La Houille Blanche. 5 112-119

[9] Faurès J.-M., Goodrich D.C., Woolhiser D.A., \& SOROOSHIAN S. (1995) - Impact of small-scale spatial rainfall variability on runoff modeling. J. Hydrol. 173 309-326

[10] SINGH V.P. (1997) — Effect of spatial and temporal variability in rainfall and watershed characteristics on stream flow hydrograph. Hydrol. Process. 11 1649-1669

[11] Arnaud P., Bouvier C., Cisneros L., \& Dominguez R. (2002) - Influence of rainfall spatial variability on flood prediction. J. Hydrol. $260216-230$

[12] Smith M.B., Koren V.I., Zhang Z., Reeda S.R., Panb J-J., \& MoredaA F. (2004) - Runoff response to spatial variability in precipitation : an analysis of observed data. J. Hydrol. $298267-$ 286

[13] Rebora N., Ferraris L., Von Hardenberg J., \& Provenzale A. (2006) - Rainfall downscaling and flood forecasting : a case study in the Mediterranean area. Nat. Hazards Earth Syst. Sci. 6 611-619

[14] Aronica G., \& Cannarozzo M. (2000) - Studying the hydrological response of urban catchments using a semi-distributed linear non-linear model. J. Hydrol. 238 35-43

[15] Segond, M-L., Wheater H.S., \& Onof C. (2007) - The significance of small-scale spatial rainfall variability on runoff modelin. J. Hydrol. 173 309-326

[16] Gires A., Onof C., Maksimovic C., Schertzer D., TChiguirinsKaiA I., \& Simoes N. (2011) - Quantifying the impact of small scale unmeasured rainfall variability on urban hydrology through multifractal downscaling: a case study. Soumis dans J. Hydrol.

[17] Harrison D.L., Driscoll S.J., \& Kitchen M. (2000) Improving precipitation estimates from weather radar using quality control and correction techniques. Met Appl. 7(2) 135144
[18] Lavallée D., Lovejoy S., \& Ladoy P. (1993) - Nonlinear variability and landscape topography: analysis and simulation. Fractals in geography, L. De Cola and N. Lam Eds. PrenticeHall. 171-205

[19] Marsan D., Schertzer D., \& Lovejoy S. (1996) - Causal space-time multifractal processes: Predictability and forecasting of rain fields. J. Geophys. Res. 101(26) 333-326-346

[20] DEIDDA R. (2000) - Rainfall downscaling in a space-time multifractal framework. Water Resour. Res. 36 1779-1794

[21] Gires A., Tchiguirinskaia I., Schertzer D., \& Lovejoy S. (2011) — Analyses multifractales et spatio-temporelles des précipitations du modèle Méso-NH et des données radar. Hydrol. Sci. J. 56(3) 380-396

[22] Venugopal V., Foufoula Georgiou E., \& Sapozhnikov V. (1999) - A space-time downscaling model for rainfall. J. Geophys. Res. 104 (D16) 19705-19721

[23] Olsson J., Uvo C.B., \& JinNo K. (2001) - Statistical atmospheric downscaling of short-term extreme rainfall by neural networks. Phys. Chem. Earth (B) 26(9) 695-700

[24] Ferraris L., Gabellani S., Rebora N., \& Provenzale A. (2003) - A comparison of stochastic models for spatial rainfall downscaling. Water Resour. Res. 39 1368-1384

[25] Rebora N., Ferraris L., Von Hardenberg J., \& Provenzale A. (2006) - The RainFARM : Downscaling LAM predictions by a Filtered AutoRegressive Model. J. Hydromet. 7 724-737

[26] Biaou A., Hubert P., Schertzer D., Tchiguirinskaia I., \& BendJoudi H. (2003) - Fractals, multifractals et prévision des précipitations. Sud Sciences et Technologies. 10 10-15

[27] Macor J., Schertzer D., \& Lovejoy S. (2007) - Multifractal methods applied to rain forecast using radar data $\mid$ [Méthodes multifractales appliquées à la prévision de pluie en utilisant des données radar. Houille Blanche. 4 92-98

[28] Thames Water Utilities Ltd Engineering (2002) Surface Water Model Of CranBrook And Seven Kings Water For London Borough Of Redbridge Appendix B, Model Development Report.

[29] Wallingford SofTware (2009) - Infoworks CS Help documentation.

[30] Maksimović, Č, Prodanović D., Boonya-Aroonnet S., Leitão J. P., Djordjević S., \& Allitt R. (2009) - Overland flow and pathway analysis for modelling of urban pluvial flooding. Journal of Hydraulic Research. 47 (4) 512-523

[31] El Tabach E., Tchiguirinskaia I., Mahmood O., \& Schertzer D. (2009) - Multi-Hydro : a spatially distributed numerical model to assess and manage runoff processes in peri-urban watersheds. Proc. Final conference of the COST Action C22 Urban Flood Management, Paris 26/27.11.2009, France. 